\title{
Preoperative medical treatment in Cushing's syndrome: frequency of use and its impact on postoperative assessment: data from ERCUSYN
}

\author{
Elena Valassi ${ }^{1}$, Holger Franz ${ }^{2}$, Thierry Brue ${ }^{3,4}$, Richard A Feelders ${ }^{5}$, Romana Netea-Maier ${ }^{6}$, Stylianos Tsagarakis' $^{7}$ \\ Susan M Webb', Maria Yaneva ${ }^{8}$, Martin Reincke9 ${ }^{1}$, Michael Droste ${ }^{10}$, Irina Komerdus ${ }^{11}$, Dominique Maiter ${ }^{12}$, \\ Darko Kastelan ${ }^{13}$, Philippe Chanson ${ }^{14,15,16}$, Marija Pfeifer ${ }^{17}$, Christian J Strasburger ${ }^{18}$, Miklós Tóth ${ }^{19}$, \\ Olivier Chabre ${ }^{20}$, Michal Krsek21, Carmen Fajardo22, Marek Bolanowski' ${ }^{23}$, Alicia Santos ${ }^{1}$, Peter J Trainer ${ }^{24}$, \\ John A H Wass ${ }^{25}$ and Antoine Tabarin ${ }^{26}$ for the ERCUSYN Study Group ${ }^{\dagger}$
}

\begin{abstract}
${ }^{1}$ IIB-Sant Pau and Department of Endocrinology/Medicine, Hospital Sant Pau, UAB, and Centro de Investigación Biomédica en Red de Enfermedades Raras (CIBER-ER, Unidad 747), ISCIII, Barcelona, Spain, ²Lohmann \& Birkner Health Care Consulting GmbH, Berlin, Germany, ${ }^{3}$ Aix-Marseille Université, CNRS, CRN2M UMR 7286, Marseille, France, ${ }^{4}$ APHM, Hôpital Conception, Marseille, France,

${ }^{5}$ Erasmus University Medical Centre, Rotterdam, The Netherlands, ${ }^{6}$ Radboud University Medical Centre, Nijmegen, The Netherlands, ${ }^{7}$ Athens Polyclinic General Hospital, Evangelismos Hospital, Athens, Greece, ${ }^{8}$ Medical University of Sofia, Sofia, Bulgary, ${ }^{9}$ Medizinische Klinik und Poliklinik IV, Campus Innestadt, Klinikum der Universität München, München, Germany, ${ }^{10}$ Praxis für Endokrinologie Droste, Oldenburg, Germany, ${ }^{11}$ Moscow Regional Research Clinical Institute n.a. Vladimirsky, Moscow, Russia, ${ }^{12}$ UCL Cliniques Universitaires St Luc, Brussels, Belgium, ${ }^{13}$ Department of Endocrinology, University Hospital Zagreb, School of Medicine University of Zagreb, Zagreb, Croatia, ${ }^{14}$ Univ Paris-Sud, Université Paris-Saclay UMR-S1185, Paris, France, ${ }^{15}$ Assistance Publique-Hôpitaux de Paris, Hôpital de Bicêtre, Service de Endocrinologie et des Maladies de la Reproduction, Paris, France, ${ }^{16}$ Institut National de la Santé et de la Recherche Médicale U1185, Paris, France, ${ }^{17}$ Department of Endocrinology, University Medical Centre Ljubljana, Ljubljana Slovenia, ${ }^{18}$ Division of Clinical Endocrinology, Department of Medicine CCM, Charité-Universitätsmedizin, Berlin, Germany, ${ }^{19} 2$ nd Department of Medicine, Semmelweis University, Budapest, Hungary, ${ }^{20}$ Service d'Endocrinologie-Diabétologie-Nutrition, Grenoble Cedex, France, ${ }^{21} 2$ nd Department of Medicine, 3rd Faculty of Medicine, Charles University and University Hospital Kralovske Vinohrady, Prague, Czech Republic, ${ }^{22}$ Department of Endocrinology, Hospital Universitario de la Ribera, Alzira, Spain, ${ }^{23}$ Department of Endocrinology, Diabetology and Isotope Therapy, Wroclaw Medical University, Wroclaw, Poland, ${ }^{24}$ Department of Endocrinology, Christie Hospital, Manchester, UK, ${ }^{25}$ Oxford University Hospital Foundation Trust, Oxford, UK, and ${ }^{26}$ Centre Hospitalier Universitaire de Bordeaux, Bordeaux, France
\end{abstract}

Correspondence should be addressed to S M Webb Email swebb@santpau.cat

\begin{abstract}
Background: Surgery is the definitive treatment of Cushing's syndrome (CS) but medications may also be used as a first-line therapy. Whether preoperative medical treatment (PMT) affects postoperative outcome remains controversial.

Objective: (1) Evaluate how frequently PMT is given to CS patients across Europe; (2) examine differences in preoperative characteristics of patients who receive PMT and those who undergo primary surgery and (3) determine if PMT influences postoperative outcome in pituitary-dependent CS (PIT-CS).

Patients and methods: 1143 CS patients entered into the ERCUSYN database from 57 centers in 26 countries. Sixtynine percent had PIT-CS, 25\% adrenal-dependent CS (ADR-CS), 5\% CS from an ectopic source (ECT-CS) and $1 \%$ were classified as having CS from other causes (OTH-CS).

Results: Twenty per cent of patients took PMT. ECT-CS and PIT-CS were more likely to receive PMT compared to ADR-CS $(P<0.001)$. Most commonly used drugs were ketoconazole $(62 \%)$, metyrapone $(16 \%)$ and a combination of both (12\%). Median (interquartile range) duration of PMT was 109 (98) days. PIT-CS patients treated with PMT had more severe clinical features at diagnosis and poorer quality of life compared to those undergoing primary surgery (SX) $(P<0.05)$. Within 7 days of surgery, PIT-CS patients treated with PMT were more likely to have normal cortisol
\end{abstract}

www.eje-online.org

https://doi.org/10.1530/EJE-17-0997
C) 2018 European Society of Endocrinology Printed in Great Britain
Published by Bioscientifica Ltd. 
$(P<0.01)$ and a lower remission rate $(P<0.01)$. Within 6 months of surgery, no differences in morbidity or remission rates were observed between SX and PMT groups.

Conclusions: PMT may confound the interpretation of immediate postoperative outcome. Follow-up is recommended to definitely evaluate surgical results.

European Journal of

\section{Introduction}

Surgery is the first-line treatment in patients with Cushing's syndrome (CS), but preoperative medical therapy (PMT) is often given, in order to control cortisol excess and correct severe comorbidities prior to the surgical procedure $(1,2)$. PMT may also be used in those centers where, for any reasons (e.g. long waiting time for surgery, limited availability of a specialized surgeon, etc.), surgical intervention cannot be performed soon after diagnosis of CS (2).

It has been hypothesized that patients with Cushing's disease (CD) preoperatively treated with adrenal-directed medications (i.e. steroidogenesis inhibitors) might have a lower risk of intraoperative bleeding, and therefore, a better surgical outcome but no firm data confirm this hypothesis $(1,3)$. Clinical observation suggests that PMT may protect CD patients from the occurrence of hypoadrenalism in the immediate postsurgical period, an important information that is actually considered as the gold standard to diagnose remission of the disease $(4,5)$. However, PMT may also confound the correct interpretation of outcome early after transsphenoidal surgery due to the 'de-repression' of the normal corticotroph cells in the remaining pituitary gland $(4,6)$. As a matter of fact, data on the potential, beneficial impact of PMT on perioperative morbidity and the immediate surgical and long-term outcomes in CD patients are scant and inconclusive $(3,7,8)$.

The ERCUSYN is the largest prospective database existing to date which collects information on diagnosis, management and long-term follow-up in CS (9). Because ERCUSYN includes data from 57 centers in 26 European countries, it reflects the 'real-life' clinical practice and allows ascertaining whether and how PMT is used before surgery across Europe.

The aims of this study where to (1) evaluate the prevalence of preoperative medical treatment (PMT) in CS patients throughout Europe; (2) determine any differences in preoperative characteristics of patients who receive PMT and those who undergo surgery directly (SX) and
(3) determine if PMT influences postoperative outcome in patients with pituitary-dependent CS (PIT-CS).

\section{Patients and methods}

\section{Description of the database}

One-thousand three hundred forty-one patients with Cushing's syndrome (CS), included into the ERCUSYN database between January 1, 2000 and January 31, 2016, were analyzed (10). Patients were classified into four major groups: pituitary-dependent CS (PIT-CS), adrenaldependent (ADR-CS; adrenal adenoma), CS from an ectopic source (ECT-CS) and CS from other etiologies (OTH-CS).

A detailed description of the database layout has been provided elsewhere (9). This study has interrogated data entered in the 'Diagnosis', 'Therapy' and 'First follow-up visit' sections.

The 'Diagnosis' section contains information at diagnosis: baseline demographic and anthropometric characteristics, etiology of CS and diagnosis date, delay between onset of symptoms and final diagnosis of CS, other specialists consulted for Cushing's symptoms before correct diagnosis, clinical features, comorbidities, bone status and two questionnaires on quality of life (CushingQoL and EuroQoL-5D). It also contains a subsection comprising diagnostic tests; for each of them, hormone concentrations, units and diagnostic interpretation ('supporting' or 'not supporting' the diagnosis) is required.

The 'Therapies' section includes specific information on any treatment received (medical, surgery, radiotherapy). In the 'medical treatment' section, participants are asked to enter information on any medication specifically used to treat hypercortisolism before surgery, including ketoconazole, metyrapone, cabergoline, mitotane, mifepristone, etomidate and aminogluthetimide. Starting and final date of medical treatment as well as starting and final dose of each drug is also required. 
The 'Surgery' section contains information on date and type of procedure, histology report and the clinical/ biochemical outcome in the immediate, postoperative period. In particular, participants are requested to indicate, when available, the clinical status early (within 7 days) after surgery, by choosing between the options 'no benefit' or 'cure/remission'. Parameters on which outcome judgment was made were also asked for, namely 'serum/urine cortisol' ('low', 'normal', 'high'), 'clinical improvement' ('yes', 'no') and 'need for glucocorticoids' ('yes', 'no'). 'Low' cortisol levels refer to those values, which are below the lowest limit of the normal range on the assay used in each center. 'Normal' cortisol levels refer to values within the normal range. 'Clinical improvement' refers to recovery characteristics early within the first week - after surgery (subjective well-being of patients, and prevalence of perioperative morbidity). The 'Follow-up visit' section contains several biochemical testing, including morning serum cortisol and overnight 1-mg dexamethasone suppression test (DST). Centers are asked to provide information on both the value of hormone measurement and its diagnostic interpretation, i.e., 'low', 'normal', 'high', according to whether the value is below, within or above the normal range of the assay used in each center. In this study, the cut-offs of $5 \mu \mathrm{g} /$ dL $(138 \mathrm{nmol} / \mathrm{L})$ has been used for both morning serum cortisol and serum cortisol after an overnight DST (5).

Glucocorticoid replacement was withdrawn at least $24 \mathrm{~h}$ prior to cortisol evaluation at any time points. The patient's surgical outcome ('active hypercortisolism' or 'remission/cure') assessed at first visit after surgery is also collected. 'First visit after surgery' refers to the first visit performed after 7 and up to 180 days after surgical intervention $(7,11)$. In this time frame, patients did not receive any additional treatment for CS. Comorbidities are also collected, including hypertension, thromboembolism, hypopituitarism, dyslipidaemia, diabetes mellitus, cardiovascular diseases, osteoporosis, fractures and depression.

If a specific item is not available, participants are asked to select 'not answered' (i.e. when information was missing) or 'not known' (when a test or clinical evaluation had been performed but results were not available for any reason).

The ERCUSYN study was approved by the ethics committee (CEIC) of the Hospital Sant Pau, Barcelona, Spain, which is the coordinating center. In addition, the local ethics committee approval was obtained for each participating institution and all patients gave their written informed consent, depending on national legal requirements.
All the data reported into the system were carefully monitored for inconsistencies, queried when necessary and validated before statistical analysis.

\section{Statistical analysis}

SPSS for Windows, version 22.0 (SPSS) was used to perform data analysis. Data on blood pressure and QoL scores are expressed as mean \pm s.D., all the other values, not normally distributed, are expressed as median (interquartile range, IQR). Comparison between two groups was carried out using the Mann-Whitney's $U$ test or the Student's $t$-test (depending on data distribution) for quantitative variables, and the $\chi^{2}$ for categorical variables. The diagnostic performance of the tests was compared calculating the likelihood ratio from contingency tables; significance was identified using the adjusted residual. Stepwise logistic regression was used to identify clinical variables and type of treatment - PMT or SX - that might predict cortisol levels and outcome in the immediate -7 days postoperative period as well as at first postoperative visit within 180 days of surgery, in PIT-CS patients. Statistical significance was defined as a two-tailed $P$ value $<0.05$.

\section{Results}

\section{General characteristics of the overall population}

Of 1341 patients included, 904 (67\%) had PIT-CS, 335 (25\%) had ADR-CS, 80 (6\%) had ECT-CS and $22(2 \%)$ had OTH-CS. Specific etiologic information has been described in detail elsewhere (10). Briefly, 79\% of patients were women, median age was 44 years (IQR, 21 years), and median time elapsed from clinical onset to diagnosis was 2 years (IQR, 3 years) (10).

\section{First-line treatment}

Of 1341 patients included, 1143 had information available on treatment (85\%). Eight hundred and ninetythree $(78 \%)$ underwent surgery without receiving prior medical treatment (SX group), while 226 (20\%) took presurgical medical treatment (PMT group). Twenty-four (2\%) additional patients received medical treatment only, without subsequent intervention and, therefore, were excluded from the analysis. Distribution of each modality of treatment according to CS etiology is shown in Table 1 .

ADR-CS patients were more likely to undergo surgery as a first-line treatment in comparison to both PIT-CS and 
Table 1 Distribution of treatment modalities based on CS etiology.

\begin{tabular}{l} 
Treatment group \\
\hline Only surgery (SX) \\
Presurgical medical treatment (PMT) \\
Only medical treatment (MT) \\
Overall
\end{tabular}

\begin{tabular}{c}
\hline PIT-CS \\
\hline $583(74)$ \\
$184(23) * *$ \\
$20(3)$ \\
787 \\
\hline
\end{tabular}

\begin{tabular}{c}
\hline ADR-CS \\
\hline $262(93)^{*}$ \\
$19(7)$ \\
0 \\
281
\end{tabular}

\begin{tabular}{c}
\hline ECT-CS \\
\hline $33(56)$ \\
$22(37)^{* *}$ \\
$4(7)^{*}$ \\
59
\end{tabular}

\begin{tabular}{c}
\hline OтH-CS \\
\hline $15(94)$ \\
$1(6)$ \\
0 \\
16 \\
\hline
\end{tabular}

\begin{tabular}{c}
\hline Overall \\
\hline $893(78)$ \\
$226(20)$ \\
$24(2)$ \\
1143 \\
\hline
\end{tabular}

Percentages are shown in parenthesis.

* $P<0.001$ vs PIT-CS and ECT-CS; ** $P<0.001$ vs ADR-CS.

ADR-CS, adrenal-dependent CS; CS, Cushing's syndrome; ECT-CS, CS from an ectopic source; OTH-CS, CS from other etiologies; PIT-CS, pituitary-dependent CS.

ECT-CS (262/281 (93\%) for ADR-CS vs 583/787 (74\%) for PIT-CS and 33/59 (56\%) for ECT-CS; $P<0.001)$. Both PIT-CS (184/787 (23\%) and ECT-CS patients (22/59 (37\%)) were more likely to receive PMT compared to ADR-CS $(19 / 281$ (7\%); $P<0.001)$ (Table 1). Countries where PMT was more frequently used in PIT-CS patients were the Netherlands (52\%), Spain (41\%), UK (39\%), Russia (27\%) and France (15\%).

\section{Characteristics of PMT: type, dose and duration}

Two hundred and twenty-six CS patients (20\%) were treated with PMT. Data on dose and duration of each treatment modalities are shown in Table 2. Most patients $(142 / 226(63 \%))$ were preoperatively treated with KTZ alone, of whom $80 \%$ were PIT-CS, $11 \%$ ECT-CS and $9 \%$ ADR-CS. MTP alone was administered to 36 patients (16\%), of whom $85 \%$ had PIT-CS, 9\% ADR-CS and 6\% ECT-CS. All 14 (6\%) patients taking CAB had PIT-CS. MIF was taken by five patients, 4 with ECT-CS and 1 with PIT-CS.

The combination of KTZ and MTP was used in 28 (12\%) patients, of whom $82 \%$ had PIT-CS, 11\% ADR-CS and 7\% ECT-CS. Combination of KTZ and CAB was administered to 12 (5\%) patients, most with PIT-CS (92\%). The combination of KTZ and MIF was taken by 5 patients, 3 with PIT-CS and 2 with ECT-CS. Combination of KTZ and either mitotane or aminoglutethimide was used in 2 PIT-CS patients. CAB or mitotane was added to a combination of KTZ and MTP in 2 PIT-CS patients.

Median (IQR) duration of PMT was 107 (98) days. No differences in maximum dose, mean dose and duration of treatment for any of the medications were found between the etiologic groups.

\section{Preoperative characteristics}

PIT-CS patients who received PMT had worse baseline clinical features as compared with those in the SX group (Fig. 1). In particular, the PMT group had higher mean $( \pm$ S.D.) systolic and diastolic blood pressure than the SX group $(145 \pm 21$ vs $139 \pm 21 \mathrm{mmHg}$ for systolic, $P=0.001$; $90 \pm 15$ vs $86 \pm 14 \mathrm{mmHg}$ for diastolic, $P=0.002$ ) (Fig. $1 \mathrm{~A}$ ). Prevalence of some hypercortisolism-related characteristics was greater in the PMT than in the SX group, including skin alterations (easy bruising, purple striae) (145/173 (84\%)

Table 2 Type and dose of medications (single or in combination), and duration of medical treatment in 226 patients treated prior to surgery.

\begin{tabular}{|c|c|c|}
\hline Type & $\boldsymbol{n}(\%)$ & Maximum dose (mg/day)* \\
\hline \multicolumn{3}{|c|}{ Single medication } \\
\hline KTZ & $142(63)$ & $600(200)$ \\
\hline MTP & $36(16)$ & $1250(1250)$ \\
\hline$C A B$ & $14(6)$ & $1(0.65)$ \\
\hline MIF & $5(2)$ & $600(100)$ \\
\hline \multicolumn{3}{|c|}{ Combined medication } \\
\hline \multirow[t]{2}{*}{$\mathrm{KTZ}+\mathrm{MTP}$} & $28(12)$ & KTZ 600 (400) \\
\hline & & MTP 1500 (1250) \\
\hline \multirow[t]{2}{*}{$\mathrm{KTZ}+\mathrm{CAB}$} & $12(5)$ & KTZ 700 (350) \\
\hline & & CAB $0.5(0.5)$ \\
\hline \multirow[t]{2}{*}{$\mathrm{KTZ}+\mathrm{MIF}$} & $5(2)$ & KTZ 800 (400) \\
\hline & & MIF 400 (200) \\
\hline
\end{tabular}

\begin{tabular}{c}
\hline Mean dose $(\mathbf{m g} / \text { day })^{*}$ \\
\hline $600(200)$ \\
$1062(917)$ \\
$0.8(0.5)$ \\
$600(100)$
\end{tabular}

KTZ 600 (275) MTP 1453 (750)

KTZ 650 (350)

CAB $0.5(0.5)$

KTZ $800(400)$

MIF $400(100)$

\begin{tabular}{c} 
Duration (days) \\
\hline $97(82)$ \\
$114(129)$ \\
$109(193)$ \\
$42(188)$
\end{tabular}

\begin{tabular}{c}
\hline CUD (g)** \\
\hline $51(64)$ \\
$104(195)$ \\
$96(224)$ \\
$34(112)$
\end{tabular}

KTZ 88 (145) MTP 101 (200)

KTZ $113(258)$

CAB 93 (54)

KTZ 109 (311)

MIF 35 (373)
KTZ 37 (82)

MTP 126 (204)

KTZ 43 (157)

CAB 47 (146)

KTZ 108 (243)
MIF $14(221)$

Doses and duration are shown as median (interquartile range, IQR).

*Dose for cabergoline (CAB) is expressed as mg/week; * ${ }^{*}$ CUD for CAB is expressed as mg.

$\mathrm{CAB}$, cabergoline; CUD, cumulative dose; KTZ, ketoconazole; MIF, mifepristone; MTP, metyrapone. 
A

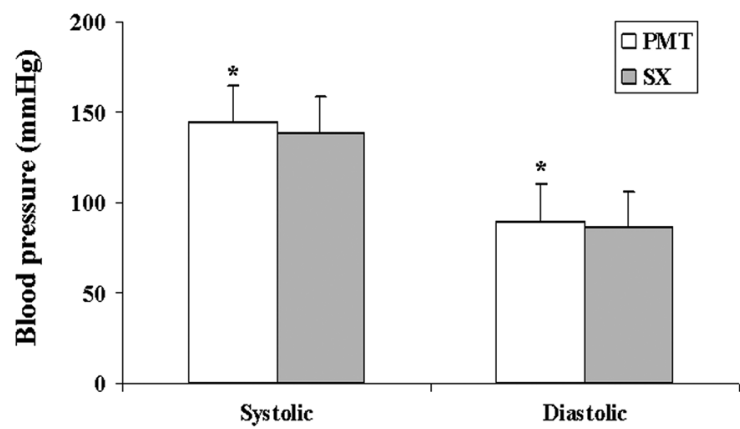

B

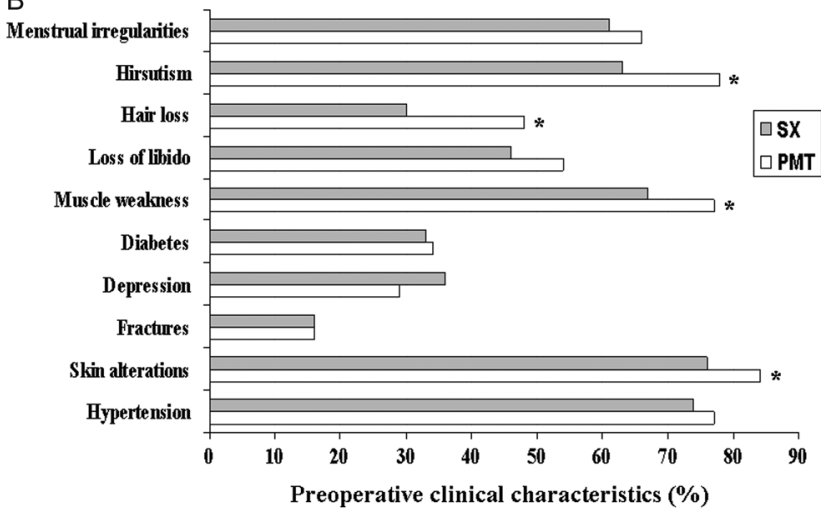

C

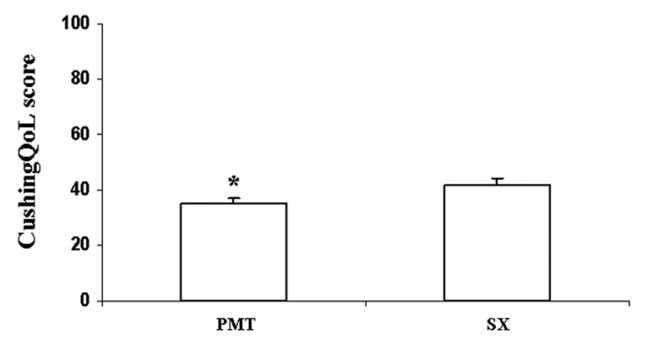

\section{Figure 1}

Preoperative clinical characteristics in PIT-CS patients who received presurgical medical treatment (PMT) and PIT-CS patients who were operated on without prior medical therapy (SX). (A) Systolic and diastolic blood pressure in PMT vs SX patients. ${ }^{*} P<0.01$, indicating more elevated systolic and diastolic blood pressure in PMT vs SX $(145 \pm 21$ vs $139 \pm 21 \mathrm{mmHg}$ and $90 \pm 15$ vs $86 \pm 14 \mathrm{mmHg}$, respectively). (B) Clinical features in PMT vs SX patients. ${ }^{*} P<0.05$, indicating greater prevalence of hypercortisolism-related features in PMT as compared with SX. (C) Quality of life (QoL), as measured using the CushingQoL questionnaire, in PMT vs $S X$ patients. ${ }^{*} P=0.035$, indicating worse QoL in PMT than $\mathrm{SX}$ (35.6 \pm 17.3 vs $42 \pm 1$, respectively).

vs $412 / 544$ (76\%); $P=0.026)$, muscle atrophy/weakness (126/163 (77\%) vs 331/497 (67\%); $P=0.010)$, hair loss (in women) $(46 / 96$ (48\%) vs $100 / 334(30 \%) ; P=0.001)$ and hirsutism (in women) (92/118 (78\%) vs 260/411 (63\%); $P=0.003$ ) (Fig. 1B). Mean ( \pm S.D.) CushingQoL score was
Table 3 Pituitary imaging in 688 PIT-CS patients receiving either PMT or SX.

\begin{tabular}{|c|c|c|c|}
\hline MRI finding & PMT $(n=171)$ & $\mathbf{S X}(n=517)$ & $P$ value \\
\hline Not seen & $51(30)$ & $103(20)$ & 0.031 \\
\hline Microadenoma & $84(57)$ & 295 (49) & 0.093 \\
\hline Macroadenoma & $36(23)$ & $119(21)$ & 0.67 \\
\hline
\end{tabular}

Percentages are expressed in parenthesis.

MRI, magnetic resonance imaging; PMT, preoperative medical therapy; SX, surgery.

also lower in the PMT than the SX group, consistent with poorer quality of life in the former (36 \pm 1 vs $42 \pm 17$, respectively; $P=0.035$ ) (Fig. $1 \mathrm{C}$ ).

Data on pituitary imaging in PMT vs SX patients are shown in Table 3. PMT patients (51 of $171(30 \%)$ ) were more likely to have a negative MRI compared to SX patients (103 of $517(20 \%))(P=0.031)$.

No differences in the preoperative characteristics were found in the other etiologic groups.

\section{Immediate postoperative evaluation in PIT-CS patients treated with steroidogenesis inhibitors as PMT}

In the immediate postoperative period (within 7 days of surgery), 'cure' or 'remission', as defined by the ERCUSYN partners, was more frequently reported in PIT-CS patients who underwent SX as the only treatment as compared with patients who took steroidogenesis inhibitors (ketoconazole, metyrapone, mitotane) (344/459 (75\%) vs $108 / 176(61 \%) ; P=0.003)$. Patients who received SX were more likely to be classified as being in remission as compared with those who took PMT (OR 1.86 (95\% CI 1.16-2.96); $P=0.009$ ). This significance remained after adjusting for preoperative features (skin alterations and muscle weakness), adenoma size, visualization of a pituitary adenoma on MRI and histology confirmation of an adenoma $(P=0.046)$.

As expected, PIT-CS patients who received PMT were more frequently reported to have cortisol levels within the 'normal' range as compared with the SX group (40/176 (23\%) vs 56/448 (13\%); $P=0.006)$. On the other hand, low or undetectable cortisol levels were more frequently reported in SX patients as compared with PMT patients (307/448 (69\%) vs 106/176 (60\%); $P=0.010)$.

SX patients were more likely to have low/undetectable cortisol levels early after surgery compared to the PMT group (OR 0.48 (95\% CI $0.30-0.76$ ); $P=0.002$ ). This significance remained after adjusting for preoperative features (skin alterations and muscle weakness), adenoma 
size, visualization of a pituitary adenoma on MRI and histology confirmation of an adenoma $(P=0.017)$.

Postoperative clinical improvement was more frequently reported in SX than in PMT patients (351/423 $(83 \%)$ vs $123 / 161(76 \%)(P=0.040))$.

\section{First postoperative evaluation within 180 days of surgery in PIT-CS patients treated with steroidogenesis inhibitors as PMT}

The first postoperative visit after discharge from the hospital ( $>7$ days after surgery) was performed within the selected period of 180 days in 723 patients. Mean ( \pm S.D.) time for this visit was $96.3 \pm 84.2$ days. In comparison to the observations within the first week after surgery, no difference in the prevalence of remission was found between SX (508/548 (93\%)) and PMT (156/174 (90\%)) patients; $P=0.21$. Prevalence of cortisol elevation above $5 \mu \mathrm{g} / \mathrm{dL}(138 \mathrm{nmol} / \mathrm{L})$ after overnight $1-\mathrm{mg}$ DST was slightly greater in the PMT than in the SX (19/30 (63\%) vs $21 / 72(29 \%) ; P=0.048)$. When the cut-off of $1.8 \mu \mathrm{g} / \mathrm{dL}$ $(50 \mathrm{nmol} / \mathrm{L})$ was evaluated, no differences in the responsiveness of cortisol were observed between the two groups.

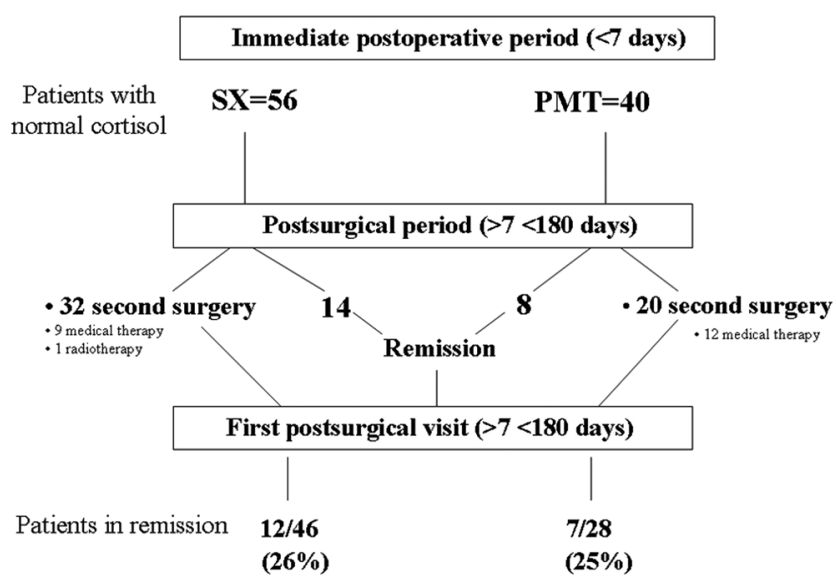

\section{Figure 2}

Outcome in 96 patients who had 'normal' cortisol levels in the immediate postoperative period ( $<7$ days), within 180 postsurgical days. SX, surgery; PMT, preoperative medical treatment. To calculate the remission rate in each group, patients who underwent second-line medical therapy or radiotherapy (10 patients in the SX and 12 in the PMT group) were excluded. No differences were found in the rate of patients who underwent further treatment as well as in the remission rate between SX and PMT group $(P=0.27$ and $P=0.81$, respectively).
No differences in the prevalence of postsurgical morbidities, including thromboembolism and hypopituitarism, were observed at first post-discharge evaluation, between the PMT and SX groups.

When patients with normal cortisol levels immediately after the intervention were analyzed, 42 of 56 (75\%)in the SX group vs 32 of $40(80 \%)$ PMT patients $(P=0.27)$ received further treatment within 180 postoperative days. Remission rate at first postsurgical visit was similar in both groups (26\% for SX vs 25\% for PMT; $P=0.81$ ) (Fig. 2).

\section{Relationship between dose or duration of PMT and immediate postoperative outcome}

A relationship between dose/duration of PMT and postoperative outcome could only be found for MTP. Thirty-six PIT-CS patients were treated with MTP alone and twenty-eight with MTP in combination with other drugs.

Median (IQR) cumulative dose (CUD) of MTP was significantly greater in patients with cortisol levels within the normal range ( $n=21$ with data available) as compared with those having hypocortisolism $(n=29)$ in the immediate postoperative period (223 (269) vs 72 (141) g; $P=0.017$ ). Similarly, patients classified as being surgical failures $(n=18)$ received greater CUD of MTP as compared with those in remission $(n=34)(223$ (210) vs 95 (138) g; $P=0.026$ ), which might be a reflection of more severe disease.

\section{Perioperative mortality}

In the whole series, perioperative mortality (within one month of surgery) was not different in SX as compared with PMT group. Of 7 patients who died within one month of surgery, $4(57 \%)$ received SX as a first-line treatment and the remaining three $(43 \%)$ took PMT $(P=0.29)$.

\section{Discussion}

These ERCUSYN data show that in Europe, one out of every five patients with Cushing's syndrome (CS) undergoes preoperative administration of steroidogenesis inhibitors prior to surgery (mostly ketoconazole, metyrapone or a combination of both) and, to a lesser extent, dopamine agonists (e.g. cabergoline) and glucocorticoid receptor antagonists (e.g. mifepristone). However, use varies in different countries, ranging from $52 \%$ in The Netherlands to no use in nine countries and depending on the underlying cause, being more frequently prescribed in 
patients with an ectopic (ECT-CS) (37\%) or pituitary source (PIT-CS) (23\%), than in ADR-CS (7\%). Patients with more florid hypercortisolism at diagnosis and proteocatabolic signs, such as muscle atrophy and skin alterations, and more impaired quality of life, were more likely to receive PMT than those with a less aggressive clinical presentation. Thus, although we cannot exclude that some clinicians were prompted to use PMT due to long waiting time for surgery in their own center, our results suggest that most ERCUSYN participants tended to pretreat those patients with more severe hypercortisolism, attempting to control their comorbidities before undergoing the intervention.

Up to now, there is little evidence that PMT affects postsurgical outcome. On the one hand, those who defend its use argue that if hypercortisolism is treated, it will improve comorbidities and the patient's clinical condition, especially in severe disease, so that surgery is performed in more favorable conditions, potentially lowering the risk of perioperative complications $(3,6,8$, $12,13,14,15,16,17,18,19,20,21)$.

On the other hand, immediate evaluation in the first week after surgery to identify whether a patient is 'cured/ in remission', as suggested in some guidelines (5), may be affected by PMT. Compared to patients who go directly to surgery, which remains the first-choice therapeutic option to quickly correct cortisol excess in CS (5), in PMT patients, clinical/biochemical improvements will more frequently have begun before surgery, so changes that take place in the first week after surgery may be less dramatic. This seems to be the case, since patients who underwent surgery without taking PMT were more likely to be classified as being 'in remission', compared to those who took PMT, in whom further clinical improvement was less evident in the immediate postoperative period. In fact, the PMT group was more likely to have 'normal' cortisol levels in the first week after adenoma resection than their counterparts treated with surgery as a first-line therapy. For the first time, this finding supports the hypothesis that PMT may protect from cortisol suppression in the immediate postoperative period (4). Based on clinical observations, it has been suggested that a sustained cortisol normalization induced by steroidogenesis inhibitors in the preoperative period may 'de-repress' the healthy corticotroph cells in the pituitary (whose function is suppressed by longstanding hypercortisolism), thus avoiding hypoadrenalism early after surgery (4). A few, small studies have been published to date on this topic, mostly retrospective, showing similar cortisol levels in the immediate postsurgical period, between patients who reached eucortisolism after PMT with steroidogenesis inhibitors and those who did not $(7,8,22)$. Conversely, one report described postoperative cortisol suppression in 13 patients who had achieved eucortisolism after PMT, raising the hypothesis that pretreatment with adrenalblocking agents may ameliorate intraoperative conditions (e.g. reduced bleeding), facilitating total adenoma resection and postoperative ACTH deficiency (3).

Additionally, postoperative 'normalization' of cortisol is generally considered a marker of persistent disease and a solid predictor of possible recurrence, although there is not widespread agreement on this point $(22,23,24,25$, $26,27)$. Although it is likely that most of the ERCUSYN participants relied upon the occurrence of hypoadrenalism to define early remission, establishing it with certainty is not possible due to the lack of a unique, standardized method for cortisol measurement throughout the centers. However, we have found that both 'normal' cortisol levels and a lower rate of early remission within 7 days of surgery, as described qualitatively, are associated with the administration of PMT with steroidogenesis inhibitors in PIT-CS, regardless of factors previously associated with surgical outcome, including adenoma size, visualization of a pituitary adenoma on MRI and histology confirmation $(22,28,29,30)$.

Of note, lack of visualization of an adenoma was more frequently reported in PIT-CS patients who received PMT as compared with those who were primarily operated on. This suggests that, in some cases, cortisol-lowering drugs have been offered within a 'wait and see' approach, when a successful resection of a nonvisible ACTH-secreting pituitary tumor was considered difficult to achieve early after the diagnosis of hypercortisolism (29). However, time interval between diagnosis of cortisol excess and surgery was similar between the two groups, indicating that starting PMT did not 'delay' the intervention in the ERCUSYN PIT-CS patients.

Clearly, PMT is highly recommendable in cases of an occult ectopic source of ACTH, in order to treat acute and potentially life-threatening morbidities associated with severe hypercortisolism $(5,31,32)$; nevertheless, similar baseline clinical characteristics and visualization rate of an ectopic tumor in ECT-CS taking PMT vs those who underwent primary surgery were observed, possibly due to the limited sample size of ERCUSYN patients with this etiology.

It is reassuring, however, and demonstrated for the first time, that at first postoperative visit within 6 months of surgery, no differences were observed between the PMT and direct SX groups, as far as prevalence of postsurgical morbidities or remission rates. Moreover, thromboembolism 
was not more frequent in ERCUSYN patients with early postsurgical hypoadrenalism as compared with those having normal cortisol; these results are in line with previous observation that short-term medical therapy leading to biochemical remission does not improve the hypercoagulable state in $\mathrm{CD}$ patients (33). However, prevalence of thromboembolism may be underestimated in the ERCUSYN, depending upon intercentre differences in the monitoring strategy of postsurgical complications and different preventive protocols.

Clearly, many factors might influence this later postoperative outcome in PIT-CS patients, such as tumor size, invasion of local tissues, surgeon's expertise, disease duration and genetic defects, any of which might, in turn, overcome the potential impact of PMT on the occurrence of remission (2). Because cortisol dynamics have been shown to vary widely over the first 6-12 postoperative weeks, potentially leading to delayed stabilization of cortisol levels, later biochemical evaluation is essential to reliably distinguish the ultimate outcome of transsphenoidal surgery $(7,8,34)$. Our data demonstrating that PMT is a further confounding factor for the proper interpretation of early surgical outcome in PIT-CS patients has important implications for decisions regarding the timing of additional therapy. In fact, early, detectable levels of postoperative cortisol in PMT patients might be misinterpreted as reflecting surgical failure and prompt some clinicians to refer them for further treatment. Instead, our data clearly support the need for retesting and long-lasting follow-up in all PIT-CS patients undergoing TSS.

The ERCUSYN design does capture information on changes of cortisol levels in patients taking PMT, and so, we could not assess the efficacy of this therapeutic approach and quantify how many patients achieved presurgical eucortisolism with PMT. Previous studies reported that rate of control of hypercortisolism ranges from $45 \%$ to $93 \%$ in CS patients taking $\mathrm{KTZ}(3,8,13,16,18)$, and from $35 \%$ to $72 \%$ in those treated with MTP $(3,8,15,21)$, depending on the assays used to define cortisol normalization, the maximum dose reached and genetically based differences in the individual responsiveness to these agents (35). Combination of both medications was reported to correct hypercortisolism in $45 \%$ of patients (8).

Limitations of this study include lack of both quantitative data and standardized methods to assess both presurgical and immediate postoperative biochemical status. This is due to both intra- and inter-centre differences in the assays used and lack of information on the normal range for each of them. Another limitation, which strictly pertains to the multicentric nature of ERCUSYN, is the heterogeneity in both the indications to use PMT and surgical success rate among the centers. While this may bias the results, it may also increase their external validity, allowing to generalize the conclusions and reflect real-life practice. Large, prospective, single-center studies should be performed to confirm the robustness of our data. In addition, ERCUSYN does not provide information on the safety of medications used as PMT or the effectiveness of such an approach to adequately reduce cortisol levels. Finally, variable time interval from surgery to first postoperative assessment among patients may have limited the detection of postoperative morbidity.

In conclusion, our study on 1143 CS patients entered in the ERCUSYN database has shown that cortisol-lowering medications are used, as a first-line treatment, in $20 \%$ of patients across Europe. Use of adrenal-blocking agents prior to pituitary surgery is independently associated with higher rate of cortisol 'normalization' and lower probability of hypoadrenalism, and consequently lower immediate postoperative identification of remission, compared to patients who go directly to surgery. Thus, caution is warranted when interpreting immediate postoperative status of PMT patients. However, at first postoperative visit within 6 months of surgery, no differences in remission rates or morbidities were seen between PMT and SX patients. Thus, benefits of preoperative improvements of hypercortisolism and consequent comorbidities attained by PMT, that are still to be evaluated, have to be confronted with the desire of immediate postoperative assessment as a prognostic marker of patients' longer term outcome, as suggested in current guidelines (5). However, long-term follow-up and repeated testing are still necessary in all patients, treated or not with PMT, in order to detect recurrences and conclusively establish surgical outcome and the most adequate long-term management strategy for each individual patient. A randomized trial assessing simple endpoints, such as length of hospital stay, surgical impression and adverse effects of surgery, is needed to conclusively demonstrate that PMT is a valid option in patients waiting for surgical correction of hypercortisolism.

Declaration of interest

S M W received financial support, research grants, consultant or speaker fees from Ipsen, Novartis, Pfizer, HRA and Strongbridge. A T received financial support, research grants, consultant fees from Novartis and HRA Pharma. C J S has received lecture fees, consultancy remuneration or research support from HRA Pharma, Novartis and Strongbridge. T B received financial support, research grants, consultant or speaker fees from Ipsen, Novartis, Pfizer, Sandoz and Strongbridge. The authors declare 
that there is no conflict of interest that could be perceived as prejudicing the impartiality of the research reported.

Funding

ERCUSYN was set up with funding from the EU (PHP 800200) and been supported by unrestricted grants from Novartis, Ipsen, HRA and the European Society of Endocrinology.

\section{Acknowledgements}

ERCUSYN Study Group: A Ambrogio, Istituto Auxologico Italiano IRCCS, University of Milan, Italy; G Aranda, Department of Endocrinology, Hospital Clinic Barcelona, IDIBAPS, UB, Barcelona, Spain; M Arosio, Unit of Endocrine Diseases \& Diabetology, Department of Clinical Sciences and Community Health, University of Milan, Milan, Italy; M Balomenaki, Athens Polyclinic General Hospital, Evangelismos Hospital, Athens, Greece; P Beck-Peccoz, Endocrinology and Diabetology Unit, Fondazione IRCCS Ca' Granda - Ospedale Maggiore Policlinico, University of Milan, Milan, Italy; C Berr-Kirmair, Medizinische Klinik und Poliklinik IV, Campus Innestadt, Klinikum der Universität München, München, Germany; J Bollerslev, Section of Specialized Endocrinology, Oslo University Hospital, and Faculty of Medicine, University in Oslo, Oslo, Norway; D Carvalho, Hospital de San Joao, Porto, Portugal; F Cavagnini, Istituto Auxologico Italiano IRCCS, Milan, Italy; E Christ, University Hospital of Bern, Inelspital, Division of Endocrinology, Diabetology and Clinical Nutrition, Bern, Switzerland; F Demtröder Zentrum fur Endokrinologie, Diabetologie, Rheumatologie Dr Demtröder \& Kollegen im MVZ, Dr. Eberhard \& Partner und Klinikum Dortmund, Germany; J Denes, Division of Endocrinology, 2nd Department of Medicine, State Health Center, Budapest, Hungary; C Dimopoulou, Max-Planck-Gessellschaft zur Forderung der Wissenschaften e.V., Munich, Germany; A Dreval, Moscow Regional Research Clinical Institute n.a. Vladimirsky, Moscow, Russia; T Dusek, Department of Endocrinology, University Hospital Zagreb, School of Medicine University of Zagreb, Zagreb, Croatia; E Erdinc, Uludag University School of Medicine, Bursa, Turkey; J A Evang, Section of Specialized Endocrinology, Oslo University Hospital, and Faculty of Medicine, Oslo University in Oslo, Oslo, Norway; J Fazel, Medizinische Klinik und Poliklinik IV, Campus Innestadt, Klinikum der Universität München, München, Germany; S Fica, Elias Hospital, Bucharest, Romania; E Ghigo, Molinette Hospital, Department of Internal Medicine, Turin, Italy; M Goth, Division of Endocrinology, 2nd Department of Medicine, State Health Center, Budapest, Hungary; Y Greenman, Institute of Endocrinology, Metabolism and hypertension, Tel Aviv, Israel; V Greisa, Medizinische Universitat Wien, Wien, Austria; I Halperin, Department of Endocrinology, Hospital Clinic Barcelona, IDIBAPS, UB, Barcelona, Spain; FA Hanzu, Department of Endocrinology, Hospital Clinic Barcelona, IDIBAPS, UB, Barcelona, Spain; A Hermus, Radboud University Medical Center, Nijmegen The Netherlands; G Johannsson, Goteborg University, Goteborg, Sweden; P Kamenicky, Univ Paris-Sud, Université Paris-Saclay UMR-S1185, Le Kremlin Bicêtre, Paris, France, Assistance Publique-Hôpitaux de Paris, Hôpital de Bicêtre, Service de Endocrinologie et des Maladies de la Reproduction, Le Kremlin Bicêtre, Paris, Institut National de la Santé et de la Recherche Médicale U1185, Le Kremlin Bicêtre, Paris, France; A KasperlikZaluska, Centre for Postgraduate Medical Education, Warsaw, Poland; J Kirchner, Division of Clinical Endocrinology, Department of Medicine CCM, Charité-Universitätsmedizin, Berlin, Germany; I Kraljevic, Department of Endocrinology, University Hospital Zagreb, Zagreb, Croatia; A Kruszynska, Centre for Postgraduate Medical Education, Warsaw, Poland; I Lambrescu, Elias Hospital, Bucharest, Romania; S Lang, Max-Planck-Gessellschaft zur Forderung der Wissenschaften e.V., Munich, Germany; A Luger, Medizinische Universitat Wien, Wien, Austria; N Marpole, Christie Hospital, NHS Trust, Manchester, UK; S Martin, Elias Hospital, Bucharest, Romania; M Martinie, Service d'Endocrinologie-Diabétologie-Nutrition, Grenoble Cedex, France; O Moros, Zentrum fur Endokrinologie, Diabetologie, Rheumatologie Dr Demtröder \& Kollegen im MVZ Dr. Eberhard \& Partner und Klinikum Dortmund; J Newell-Price, The University of Sheffield, Sheffield, UK; M Orbetzova, Clinic of Endocrinology and Metabolic Diseases, 'Sv.Georgy' University Hospital, Medical University, Plovdiv, Bulgaria; I Paiva, Hospitais da Universidade de Coimbra; F Pecori Giraldi, Istituto Auxologico Italiano IRCCS, University of Milan, Italy; A M Pereira, Leiden University Medical Center, Leiden, The Netherlands; J Pickel, MaxPlanck-Gessellschaft zur Forderung der Wissenschaften e.V., Munich, Germany; V Pirags, Pauls Stradiņš Clinical University Hospital, University of Latvia, Riga, Latvia; O Ragnarsson, Goteborg University, Goteborg, Sweden; A D Reghina, Elias Hospital, Bucharest, Romania; P Riesgo, Neurosurgery Department, Hospital Universitario de la Ribera, Alzira, Spain; M Roberts, Christie Hospital, NHS Trust, Manchester, UK; S Roerink, Radboud University Medical Center, Nijmegen The Netherlands; O Roig, IIB-Sant Pau and Department of Endocrinology/Medicine, Hospital Sant Pau, UAB, and Centro de Investigación Biomédica en Red de Enfermedades Raras (CIBER-ER, Unidad 747), ISCIII; C Rowan, Christie Hospital, NHS Trust, Manchester, UK; P Rudenko, Estonian Endocrine Society, Tallinn, Estonia; M A Sahnoun, Aix-Marseille Université, CNRS, CRN2M UMR 7286, Cedex 15, Marseille, and APHM, Hôpital Conception, Marseille, France; J Salvador, University of Navarra, Pamplona, Spain; HA Sigurjonsdottir, Landspitali University Hospital, Reykjavik, Iceland and Faculty of Medicine, University of Iceland, Reykjavik, Iceland; T Skoric Polovina, Department of Endocrinology, University Hospital Zagreb, Zagreb, Croatia; R Smith, Oxford Radcliffe Hospitals NHS Trust, Oxford, UK; B Stachowska, Department of Endocrinology, Diabetology and Isotope Therapy Wroclaw Medical University, Wroclaw, Poland; G Stalla, Max-Planck-Gessellschaft zur Forderung der Wissenschaften e.V., Munich, Germany; J Tóke, 2nd Departement of Medicine, Semmelweis University, Budapest, Hungary; E Ubina, Division of Endocrinology, 2nd Department of Medicine, State Health Center, Budapest, Hungary; S Vinay, Christie Hospital, NHS Trust, Manchester, UK; M Wagenmakers, Radboud University Medical Center, Nijmegen The Netherlands; S Werner,Praxis für Endokrinologie Droste, Oldenburg, Germany; J Young, Univ Paris-Sud, Université Paris-Saclay UMR-S1185, Le Kremlin Bicêtre, Paris, France, Assistance PubliqueHôpitaux de Paris, Hôpital de Bicêtre, Service de Endocrinologie et des Maladies de la Reproduction, Le Kremlin Bicêtre, Paris, Institut National de la Santé et de la Recherche Médicale U1185, Le Kremlin Bicêtre, Paris, France; P Zdunowski, Centre for Postgraduate Medical Education, Warsaw, Poland; K Zopf, Division of Clinical Endocrinology, Department of Medicine CCM, Charité-Universitätsmedizin, Berlin, Germany; S Zopp, Medizinische Klinik und Poliklinik IV, Campus Innestadt, Klinikum der Universität München, München, Germany; I Zosin, Romanian Society for Endocrinology, Timisoara, Romania.

\section{References}

1 Feelders RA \& Hofland LJ. Medical treatment of Cushing's disease. Journal of Clinical Endocrinology and Metabolism 201398 425-438. (https://doi.org/10.1210/jc.2012-3126)

2 Pivonello R, De Leo M, Cozzolino A \& Colao A. The treatment of Cushing's disease. Endocrine Review 201536 386-486.

3 van den Bosch OF, Stades AM \& Zelissen PM. Increased long-term remission after adequate medical cortisol suppression therapy as presurgical treatment in Cushing's disease. Clinical Endocrinology 201480 184-190. (https://doi.org/10.1111/cen.12286)

4 Biller BMK, Grossman AB, Stewart PM, Melmed S, Bertagna X, Bertherat J, Buchfelder M, Colao A, Hermus AR, Hofland LJ et al. Treatment of adrenocorticotropin-dependent Cushing's syndrome: a consensus statement. Journal of Clinical Endocrinology and Metabolism 200893 2454-2462. (https://doi.org/10.1210/jc.2007-2734)

5 Nieman LK, Biller BMK, Findling JW, Murad MH, Newell-Price J, Savage MO \& Tabarin A. Treatment of Cushing's syndrome: an Endocrine Society Clinical Practice Guideline. Journal of Clinical Endocrinology and Metabolism 2015100 2807-2831. (https://doi. org/10.1210/jc.2015-1818) 
6 Castinetti F, Guignat L, Giraud P, Muller M, Kamenicky P, Drui D, Caron P, Luca F, Donadille B, Vantyghem MC et al. Ketoconazole in Cushing's disease: is it worth a try? Journal of Clinical Endocrinology and Metabolism 201499 1623-1630. (https://doi.org/10.1210/ jc.2013-3628)

7 Pereira AM, van Aken MO, van Dulken H, Schutte PJ, Biermasz NR, Smit JW, Roelfsema F \& Romijn JA. Long-term predictive value of postsurgical cortisol concentrations for cure and risk of recurrence in Cushing's disease. Journal of Clinical Endocrinology and Metabolism $2003885858-5864$.

8 Valassi E, Crespo I, Gich I, Rodriguez J \& Webb SM. A reappraisal of the medical therapy with steroidogenesis inhibitors in Cushing's syndrome. Clinical Endocrinology 201277 735-742. (https://doi. org/10.1111/j.1365-2265.2012.04424.x)

9 Valassi E, Santos A, Yaneva M, Toth M, Strasburger CJ, Chanson P, Wass JA, Chabre O, Pfeifer M, Feelders RA et al. The European Registry on Cushing's syndrome: 2-year experience. Baseline demographic and clinical characteristics. European Journal of Endocrinology 2011165 383-392. (https://doi.org/10.1530/ EJE-11-0272)

10 Valassi E, Franz H, Brue T, Feelders RA, Netea-Maier R, Tsagarakis S, Webb SM, Yaneva M, Reincke M, Droste M et al. Diagnostic tests for Cushing's syndrome differ from published guidelines: data from ERCUSYN. European Journal of Endocrinology 2017176 613-624.

11 Valassi E, Biller BMK, Swearingen B, Pecori-Giraldi F, Losa M, Mortini P, Hayden D, Cavagnini F \& Klibanski A. Delayed remission after transsphenoidal surgery in patients with Cushing's disease. Journal of Clinical Endocrinology and Metabolism 201095 601-610.

12 Jeffcoat WJ, Rees LH, Tomlin S, Jones AE, Edwards CRW \& Besser GM. Metyrapone in long-term management of Cushing's disease. BMJ 1977 ii 215-217. (https://doi.org/10.1136/ bmj.2.6081.215)

13 Loli P, Berselli ME \& Tagliaferri M. Use of ketoconazole in the treatment of Cushing's syndrome. Journal of Clinical Endocrinology and metabolism 198663 1365-1371. (https://doi.org/10.1210/jcem63-6-1365)

14 Tabarin A, Navarranne A, Guerin J, Corcuff JB, Parneix M \& Roger P. Use of ketoconazole in the treatment of Cushing's disease and ectopic ACTH syndrome. Clinical Endocrinology 199134 63-69. (https://doi.org/10.1111/j.1365-2265.1991.tb01737.x)

15 Verhelst JA, Trainer PJ, Howlett TA, Perry L, Rees LH, Grossman AB, Wass JA \& Besser GM. Short and long-term responses to metyrapone in the medical management of 91 patients with Cushing's syndrome. Clinical Endocrinology 199135 169-178. (https://doi. org/10.1111/j.1365-2265.1991.tb03517.x)

16 Sonino N, Boscaro M, Paoletta A, Mantero F \& Ziliotto D. Ketoconazole treatment in Cushing's syndrome: experience in 34 patients. Clinical Endocrinology 199935 347-352. (https://doi. org/10.1111/j.1365-2265.1991.tb03547.x)

17 Moncet D, Morando DJ, Pitoia F, Katz SB, Rossi MA \& Bruno OD. Ketoconazole therapy: an efficacious alternative to achieve eucortisolism in patients with Cushing's syndrome. Medicina 200767 26-31.

18 Castinetti F, Morange I, Jaquet P, Conte-Devolx B \& Brue T. Ketoconazole revisited: a preoperative or postoperative treatment in Cushing's disease. European Journal of Endocrinology 2008 158 91-99. (https://doi.org/10.1530/EJE-07-0514)

19 Godbaut A, Manavela M, Danilowicz K, Beauregard H, Bruno OD \& Lacroix A. Cabergoline monotherapy in the long-term treatment of Cushing's disease. European Journal of Endocrinology 2010163 709-716. (https://doi.org/10.1530/EJE-10-0382)

20 Baudry C, Coste J, Bou Khalil R, Silvera S, Guignat L, Guibourdenche J, Abbas H, Legmann P, Pertagna X \& Bertherat J. Efficiency and tolerance of mitotane in Cushing's disease in 76 patients from a single center. European Journal of Endocrinology 2012 167 473-481. (https://doi.org/10.1530/EJE-12-0358)
21 Daniel E, Aylwin S, Mustafa O, Ball S, Munir A, Boelaert K, Chortis V, Cuthbertson DJ, Daousi C, Rajeev SP et al. Effectiveness of metyrapone in treating Cushing's syndrome: a retrospective multicenter study in 195 patients. Journal Clinical Endocrinology and Metabolism 2015100 4146-4154. (https://doi.org/10.1210/jc.20152616)

22 Invitti C, Pecori Giraldi F, de Martin M \& Cavagnini F. Diagnosis and management of Cushing's syndrome: results of an Italian multicentre study. Study Group of the Italian Society of Endocrinology on the pathophysiology of the hypothalamicpituitary-adrenal axis. Journal of Clinical Endocrinology and Metabolism 199984 440-448.

23 Shimon I, Ram Z, Cohen ZR \& Hadani M. Transsphenoidal surgery for Cushing's disease: endocrinological follow-up monitoring of 82 patients. Neuosurgery $20025157-61$. (https://doi. org/10.1097/00006123-200207000-00008)

24 Hammer GD, Tyrrell JB, Lamborn KR, Apllebury CB, Hannegan ET, Bell S, Rahl R, Lu A \& Wilson CB. Transsphenoidal microsurgery for Cushing's disease: initial outcome and long-term results. Journal of Clinical Endocrinology and Metabolism 200489 6348-6357. (https:// doi.org/10.1210/jc.2003-032180)

25 Salenave S, Gatta B, Pecheur S, San-Galli F, Visot A, Lasjuaunias P, Roger P, Berge J, Young J. Tabarin A et al. Pituitary magnetic resonance imaging findings do not influence surgical outcome in adrenocortictropin-secreting microadenomas. Journal of Clinical Endocrinology and Metabolism 200489 3371-3376. (https://doi. org/10.1210/jc.2003-031908)

26 Atkinson AB, Kennedy A, Wiggam MI, Mc Cance DR \& Sheridan B. Long-term remission rates after pituitary surgery for Cushing's disease: the need for long-term surveillance. Clinical Endocrinology 200563 549-559. (https://doi.org/10.1111/j.13652265.2005.02380.x)

27 Patil CG, Prevedello DM, Lad SP, Vance ML, Thorner MO, Katznelson L \& Laws ER Jr. Late recurrences of Cushing's disease after initial successful transsphenoidal surgery. Journal of Clinical Endocrinology and Metabolism 200893 358-362. (https://doi. org/10.1210/jc.2007-2013)

28 Guillhaume B, Bertagna X, Thomsen M, Bricaire C, Vila-Porcile E, Olivier L, Racadot J, Derome P, Laudat MH, Girard F et al. Transshenoidal pituitary surgery for the treatment of Cushing's disease: results in 64 patients and long-term follow up studies. Journal of Clinical Endocrinology and Metabolism 198866 1056-1064. (https://doi.org/10.1210/jcem-66-5-1056)

29 Bochicchio D, Losa M \& Buchfelder M. Factors influencing the immediate and late outcome of Cushing's disease treated by transsphenoidal surgery: a retrospective study by the European Cushing's disease Survey Group. Journal of Clinical Endocrinology and Metabolism 199580 3114-3120.

30 Rees DA, Hanna FW, Davies JS, Mills RG, Vafidis J \& Scanlon MF Long-term follow-up results of transsphenoidal surgery for Cushing's disease in a single centre using strict criteria for remission. Clinical Endocrinology 200256 541-551. (https://doi.org/10.1046/j.13652265.2002.01511.x)

31 Kamenicky P, Droumaguet C, Salenave S, Blanchard A, Jublanc C, Gautier JF, Brailly-Tabard S, Leboulleux S, Schlumberger M, Baudin E et al. Mitotane, metyrapone and ketoconazole combination therapy as an alternative to rescue adrenalectomy for severe ACTHdependent Cushing's syndrome. Journal of Clinical Endocrinology and Metabolism 201196 1-9.

32 Corcuff JB, Young J, Masquefa-Giraud P, Chanson P, Baudin E \& Tabarin A. Rapid control of severe neoplastic hypercortisolism with metyrapone and ketoconazole. European Journal of Endocrinology 2015172 473-481. (https://doi.org/10.1530/ EJE-14-0913)

33 van der Pas R, de Bruin C, Leebeck FW, de Maat LP, Rijken DC, Pereira AM, Romijn JA, Netea Maier RT, Hermus AR, Zelissen PM 
et al. Incidence of venous thromboembolism in patients with Cushing's syndrome: a multicenter cohort study. Journal of Clinical Endocrinology and Metabolism 201196 3525-3532. (https://doi. org/10.1210/jc.2011-1661)

34 Rollin GA, Feereira NP, Junges M, Gross JL \& Czepielewski MA. Dynamics of serum cortisol levels after transsphenoidal surgery in a cohort of patients with Cushing's disease. Journal of Clinical
Endocrinology and Metabolism 200489 1131-1139. (https://doi. org/10.1210/jc.2003-031170)

35 Valassi E, Aulinas A, Glad CAM, Johannsson G, Ragnarsson O \& Webb SM. A common genetic variant in the CYP17A1 influences the therapeutic response to steroidogenesis inhibitors in patients with Cushing's syndrome. Clinical Endocrinology 201787 433-439. (https://doi.org/10.1111/cen.13414)

Received 29 November 2017

Revised version received 16 January 2018

Accepted 12 February 2018 